Edición Extra-Ordinaria. ISSN 2027-1034 P. p 337-344

Memorias del VII Encuentro Nacional de Experiencias en la Enseñanza de la Biología y la Educación Ambiental y II Congreso Nacional de Investigación en la Enseñanza de la Biología

\title{
LA EXPERIENCIA DE UNA CLASE INTEGRADA MEDIANTE RESOLUCIÓN DE PROBLEMAS
}

\author{
Fernanda Ortiz Rivera ${ }^{1}$ \\ Gonzalo Marín Oviedo ${ }^{2}$ \\ Zully Cuellar López $\mathrm{Mg}^{3}$
}

\section{Resumen}

Es una experiencia en la Institución educativa Tomas Cipriano de Mosquera con estudiantes del ciclo noveno en una clase de ciencias naturales sobre el sentido de la audición, teniendo en cuenta integrar la biología y la física mediante el tema del órgano de la audición con el tema del sonido, en el marco de un enfoque didáctico por resolución de problemas, en el cual la pregunta problema central que se generó a los estudiantes, producto de consultar sus intereses, fue: ¿por qué se produce la sordera? Esta pregunta se realizó al comienzo y al final de la clase, mostrando de esta manera al final buenos resultados de aprendizaje en los estudiantes, ya que fueron construyendo nociones, ideas y conceptos necesarios sobre el sonido con sus propiedades y cualidades; y la audición con las funciones y partes del oído, para poder resolver la pregunta central gracias a una secuencia didáctica de clase que construimos cuidadosamente en el que incluía experimentos, lecturas, explicaciones, materiales didácticos, entre otros, siendo así una clase muy activa y participativa, ya que el estudiante siempre fue el que llevo el papel principal de la clase.

Palabras Claves: Clase integrada, Resolución de problemas, órgano de la audición y sonido.

\section{An Integrated Class Experience By Means Of Troubleshooting}

\section{Abstract}

It is an experience in educational institution with Tomas Cipriano de Mosquera with ninth stage students in natural sciences class about the sense of hearing, taking into account integrating the biology and physical through the topic of the hearing organ with the topic of sound, as part of a teaching approach for problem solving, in which the central problem question students generated as a result of consulting their interests, was: why deafness occurs? This question was asked at the beginning and at end of student learning, thus showing good results at the end of student learning, since they were building concepts and

\footnotetext{
1 Estudiante de VIII semestre del programa de licenciatura en Ciencias Naturales, Universidad Surcolombiana. fer.2590@hotmail.com

${ }^{2}$ Estudiante de VIII semestre del programa de de licenciatura en Ciencias Naturales, Universidad Surcolombiana.gonzalomarin123@hotmail.com

${ }^{3}$ Docente de planta del programa de licenciatura en Ciencias Naturales, Universidad Surcolombiana cursosusco@gmail.com
} 
Edición Extra-Ordinaria. ISSN 2027-1034 P. p 337-344

Memorias del VII Encuentro Nacional de Experiencias en la Enseñanza de la Biología y la Educación Ambiental y II Congreso Nacional de Investigación en la Enseñanza de la Biología

ideas needed from sound withtheir propertiesand qualities; and from hearing with offunctions andparts of the ear to solve the problem thanks to a lesson plan that we carefully constructed which included experiments, lectures, explanations, instructional materials, among others, being a very active and participatory class, since the student was always the leading role of the class.

Keys Word: Integrated Class, Troubleshooting, hearing and sound organ.

\section{Introducción}

Es común ver en la educación formal la desconexión entre los saberes científicos por parte del maestro, obligando inevitablemente al estudiante a tratar las distintas materias como si fueran unidades en sí mismas. El saber aparecería así desvertebrado y atomizado, sin otorgarle al estudiante la oportunidad de entrever una visión de conjunto (Hernández y Prieto, 2000, citado por Cornejo, 2007, p. 1). La integración de las diferentes disciplinas científicas, por ejemplo las ciencias naturales pueden otorgan una visión transversalizada a los estudiantes siempre y cuando el maestro tenga una formación integral y esté dispuesto a enseñar de esa manera, sin embargo, hay diferentes formas de mostrar estas clases en el aula, una de ellas es mediante el enfoque didáctico de la resolución de problemas, ya que según Gaunche (2005, p. 4), una de las contradicciones al presentar un problema es buscar fenómenos y procesos que integran varias ciencias que conducen a consecuencias inesperadas para quien no conozca su esencia o sus nexos causales. De esta manera se tuvo la experiencia dirigida por estudiantes de licenciatura en ciencias naturales de la universidad surcolombiana con estudiantes del ciclo noveno de la institución educativa Tomas Cipriano de Mosquera de la ciudad de Neiva, donde se integró la biología y la física con el tema el sentido de la audición, en el marco de la pregunta central: ¿Cómo se produce la sordera?, de este modo se logró relacionar el órgano de la audición con el sonido. Tema que correspondía a las planeaciones propias de la institución.

Esta pregunta nos permitió abordar dificultades encontradas en la literatura relacionadas con enseñanza por resolución de problemas y la enseñanzaaprendizaje sobre el sentido de la audición.

En una publicación que hizo Guanche (2005, p. 7) en la revista iberoamericana de educación, la cual dice que los maestros tienen dificultades a la hora de realizar sus clase mediante resolución de problemas, ya que no presentan la contradicción adecuada en el momento de formular la pregunta problema a sus estudiantes, y para resolver esa dificultad expone de este modo 13 formas posibles de presentar el problema, investigadas hasta ese momento por la autora, recalcando de la misma manera que la mejora de estas aplicaciones contradictorias es mediante el entrenamiento sistemático del maestro para presentar mejor su situación problema. Teniendo en cuenta las 13 formas de 
Edición Extra-Ordinaria. ISSN 2027-1034 P. p 337-344

Memorias del VII Encuentro Nacional de Experiencias en la Enseñanza de la Biología y la Educación Ambiental y II Congreso Nacional de Investigación en la Enseñanza de la Biología

presentar la contradicción problémica escogimos la número 10 que dice: "Fenómenos y procesos que integran varias ciencias que conducen a consecuencias inesperadas para quien no conozca su esencia o sus nexos causales" ya que es muy a fin con nuestra clase porque integraremos la ciencia biológica que fundamenta el órgano como tal, y la ciencia física que fundamenta el sonido, siendo este último el que causa el estímulo o la percepción por el oído.

Como segunda investigación que se realizó en busca de las dificultades de aprendizaje en el tema que tratamos, encontramos según las deducciones que hicimos con base a las investigaciones realizadas por Banet (2000, p. 457), que encontró dificultades relacionadas con el aprendizaje de la disciplina biológica y por las investigaciones realizadas por Saura y Pro (2000, p. 409), que encontraron dificultades relacionadas con el aprendizaje de la disciplina física decimos que:

- Los estudiantes creen que el único órgano receptor de las señales eléctricas producto de los órganos de los sentidos es el cerebro, sin tener en cuenta que el cerebelo, el tronco encefálico y los nervios también son partes del sistema nervioso y participan activamente en la recepción de señales eléctricas.

- $\quad$ Además, ellos creen que si una persona u animal no tiene determinados comportamientos como el oír, entonces carecen de sistema nervioso o como dicen ellos, carecen de alguna parte del cerebro.

- $\quad$ Consideran que el sonido se propaga en el vacío; incluso mejor que en otro medio porque según ellos no hay resistencia.

- $\quad$ No entienden que en la propagación del sonido no hay propagación de masa, sino de la energía.

- $\quad$ Confunden la velocidad del sonido con la intensidad; además consideran que la velocidad del sonido no es constante sino que disminuye con la distancia.

- $\quad$ Tienen dificultades para reconocer las cualidades del sonido.

Las dificultades de aprendizaje mencionadas anteriormente fueron fundamentales para la secuencia didáctica de la clase porque de esta manera nos dimos cuenta de los errores que cometen los estudiantes al tratar de comprender el tema de la audición. Situación que puede afectarla resolución de la pregunta problema si no se tiene en cuenta, del mismo modo fue bueno adelantarnos a las posibles consecuencias que se pueden presentar en una clase. 
Edición Extra-Ordinaria. ISSN 2027-1034 P. p 337 - 344

Memorias del VII Encuentro Nacional de Experiencias en la Enseñanza de la Biología y la Educación Ambiental y II Congreso Nacional de Investigación en la Enseñanza de la Biología

Teniendo en cuenta la pregunta problema los contenidos conceptuales los organizamos en un mapa conceptual construidos por nosotros y que aparece en la imagen 1, apoyándonos de las definiciones de Quiroga (1975, p. 52), y Cheers (2008, p. 6). Esto nos dio una fundamentación teórica, y una estructura más flexible al tener una construcción jerárquica en nuestras mentes.

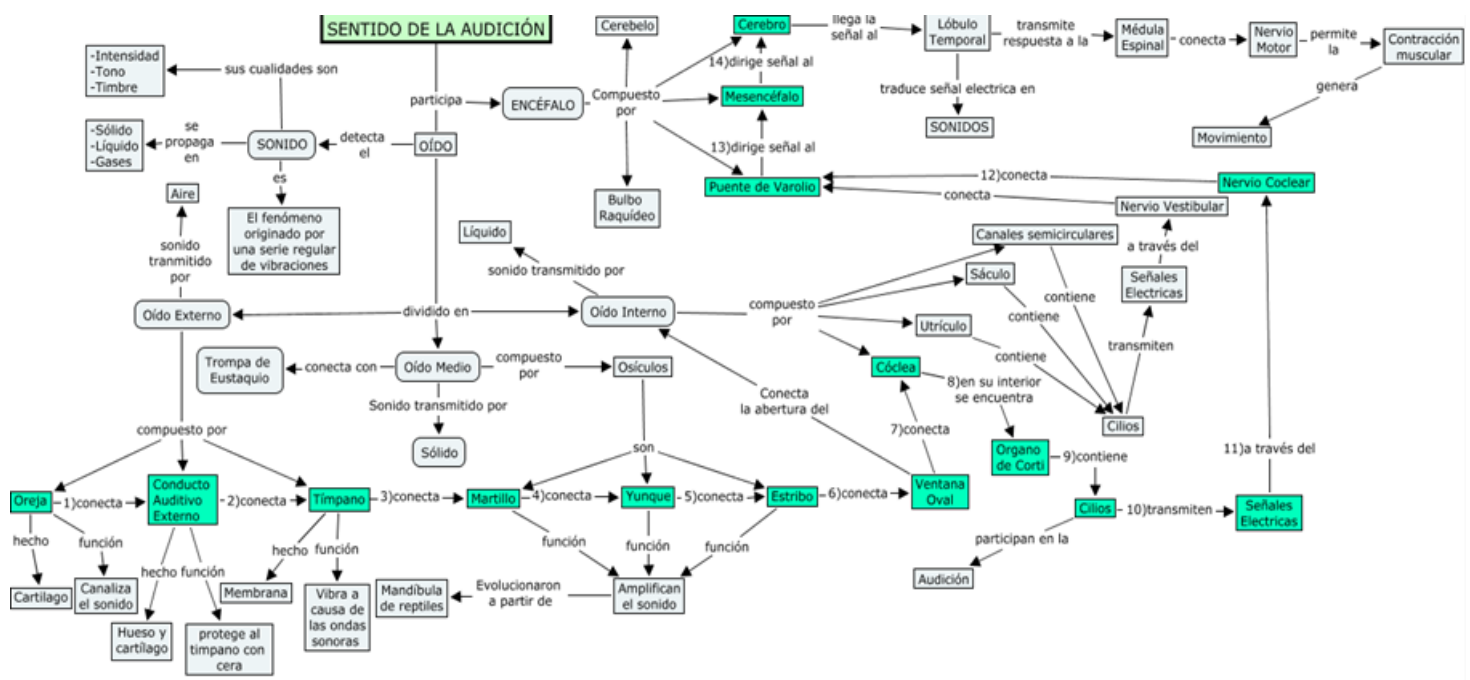

Imagen 1: Mapa conceptual sobre el sentido de la audición, la numeración de 1 a 14 en los conectores es la secuencia por donde viaja el sonido y los conceptos coloreados son los que tienen relacionan con él.

\section{Metodología}

La muestra una población vulnerable de estrato social 1 y 2, comprende un total de 21 estudiantes del ciclo noveno de la institución educativa Tomas Cipriano de Mosquera. Se propuso entonces una secuencia didáctica de clase y para su planeación tuvimos en cuenta lo siguiente:

Primeramente realizamos una encuesta dirigida a la institución y otra dirigida a los estudiantes, con el fin de conocer el contexto en el cual nos correspondió trabajar. Una vez realizadas las encuestas nos dirigimos a tabularlas y analizarlas, encontrando las ideas previas que tenían los estudiantes sobre el tema que tratamos. Esto lo pudimos deducir cuando preguntamos en la encuesta a los estudiantes, con el fin de saber qué conocían acerca del tema órganos de los sentidos. Encontramos por ejemplo que hay un mecanismo físico o biológico que permite la audición, ya que ellos preguntaron cómo es que funciona el órgano de la audición, haciéndose una idea de qué es lo que pasa al interior del oído, como si creyeran que debe de tener un complejo al menos mecánico para que se pueda percibir el sonido. Además, los estudiantes tienen también la idea de que el órgano de la audición como tal tiene un antecesor en común, ya que su pregunta fue ¿de dónde vienen esos órganos? 
Edición Extra-Ordinaria. ISSN 2027-1034 P. p 337-344

Memorias del VII Encuentro Nacional de Experiencias en la Enseñanza de la Biología y la Educación Ambiental y II Congreso Nacional de Investigación en la Enseñanza de la Biología

Después de haber conocido el contexto de la institución educativa y de los estudiantes, sus ideas previas y de haber indagado las dificultades de enseñanza y aprendizaje, continuamos a formular la pregunta problema central que fue: ¿por qué se produce la sordera? Creemos que esta pregunta estuvo bien formulada porque se fundamenta con las proposiciones de Guanche (2005 p. 4) dichas anteriormente, además el problema tiene la característica, que es abierto, es decir, con múltiples respuestas y es fácilmente observable por los estudiantes, esto les permite utilizar fácilmente esquemas que sirvan para su aprendizaje.

Con base a lo anterior se organizó y realizo una secuencia didáctica de clase con diversas actividades organizadas en tres fases que fueron: presentación de la situación problema, plan de solución y cierre, que se muestra en el cuadro 2.

Se motivó a los estudiantes éticamente mediante un ejemplo de la cotidianidad que está dirigido al respeto que se le debe de dar a las personas con sordera ya que son discriminadas comúnmente,

ción de la situación problema

Desarrollo de la clase concientizando así que el trato a ellas debe ser el mejor posible. Motivado el estudiante se generó la pregunta problema central ¿Por qué se produce la sordera?, del cual se recogieron en el tablero las hipótesis que tienen los estudiantes acerca de esta pregunta, para ser transformadas o contrastadas durante la clase.

1. Se dieron algunos fundamentos del sonido con la ayuda del análogo que es una flecha ondulada, hecha en cartulina, la cual representaba la energía.

2. Se proponen sub preguntas: ¿Qué condiciones crees que se requiere para que el sonido se propague?, luego se recogió en el tablero las hipótesis que tienen al respecto y posteriormente se realizó con los estudiantes algunos experimentos necesarios para dar solución a la pregunta.

3. Una vez los estudiantes son conscientes de que el sonido requiere de un medio para propagarse, se trabajan las cualidades del sonido a través de 4 experimentos. Durante cada experimento se plantearon preguntas como ¿Por qué uno de los objeto suena más fuerte al golpearlo?, ¿Cuándo suena más agudo el sonido y por qué? Y ¿Qué objeto produjo cada sonido? y se anotaron en el tablero las respuestas que aportaron los estudiantes.

4. Se realizó una lectura sobre la audición, para reproducir el proceso que sigue el sonido en el oído como lo decía la lectura. Esto se hizo con la ayuda de un material didáctico que es un oído a escala y la flecha en forma de onda que permitió evidenciar el recorrido, con el fin de que los estudiantes comprendieran mejor el funcionamiento del oído.

5. Se realizó otra lectura encaminada a la evolución de los osículos (martillo, yunque y estribo), comparando el odio del reptil con el del hombre y de la misma manera la mandíbula del reptil con el del 
Edición Extra-Ordinaria. ISSN 2027-1034 P. p 337-344

Memorias del VII Encuentro Nacional de Experiencias en la Enseñanza de la Biología y la Educación Ambiental y II Congreso Nacional de Investigación en la Enseñanza de la Biología

hombre, con el fin de que los estudiantes comprendan que los seres vivos no eran los mismos antes, ni serán los mismos después debido al proceso evolutivo.

6. Se resumió cómo llegamos a oír, enfatizando principalmente en señalar las partes principales del oído con su respectiva función. En esta explicación se uso un análogo para los osículos con las cajas de resonancia con pesos en sus diapasones para que el estudiante se dé cuenta que estos huesos vibran y pueden dañarse, para que tengan una comprensión mejor da la función y cuidado de estos huesos. Posteriormente se explicó con una imagen el órgano de corti.

7. Mediante otra imagen se explicó el sistema nervioso, enfatizando por dónde pasa la señal eléctrica producto de los cilios de la cóclea en el órgano de corti y dónde se traduce estas señales.

8. Se retoma la pregunta central de la clase. Entonces se escriben las respuestas de los estudiantes en el tablero, contrastándolas y analizándolas con las que se anotaron inicialmente en el tablero antes del desarrollo de la clase.

9. Los estudiantes reproducen verbalmente todo el recorrido del sonido en el oído hasta su lugar de interpretación, nombrando sus partes con la ayuda del oído a escala y la flecha ondulada.

\section{Cierre}

Se retoma el tema, apoyándonos en el mapa conceptual para recoger las ideas expresadas por los estudiantes y el profesor en el desarrollo de la secuencia didáctica de clase.

Cuadro 2. Secuencia didáctica de clase

\section{Resultados Y Discusiones}

Con relación al momento de presentación de la situación problema donde los estudiante daban sus hipótesis al contestar la pregunta problema central en la clase, dentro de sus respuestas hablaron de tres causas que producía la sordera. Entre ellas dijeron por enfermedades, por problemas de nacimiento y por lesiones. Después de implementar las actividades organizadas en la secuencia de clase se pudo apreciar durante la actividad 8 que los estudiantes explican que la sordera se producía por diferentes causas entre estas: exponerse a ruidos altos de cualquier fuente sonora provocando así daños en el oído medio; por las inflamaciones de los tejidos que recubren estos órganos no dejan vibrar los órganos como debe ser; por romperse el tímpano debido a altas presiones; también al dañarse los cilios por sonidos muy intensos no pueden vibrar más y no transmitirían señales eléctricas; y que el taponamiento del oído externo no permite que el sonido entre y así se produce la sordera. Son respuestas con un fundamento claro de los conceptos que se construyeron durante la clase en comparación con las ideas que tenían antes de desarrollar la clase, en las que seevidenciopocabase conceptual al respecto.

Estas respuestas y el recorrido del sonido hasta la interpretación de éste por el cerebro que logra explicar estudiantes en la actividad 9, indican que todas las 
Edición Extra-Ordinaria. ISSN 2027-1034 P. p 337-344

Memorias del VII Encuentro Nacional de Experiencias en la Enseñanza de la Biología y la Educación Ambiental y II Congreso Nacional de Investigación en La Enseñanza de la Biología

actividades de la secuencia didáctica de clase, que se utilizaron como los análogos, los materiales didácticos, experimentos y lecturas fueron claves para aclarar este proceso complejo que requiere de varios conceptos simultáneamente para ser interpretados de manera integral.

A medida que se dialogaba sobre los resultados de los experimentos, se generaban preguntas y se aclaraban dudas, la diversidad de actividades durante la clasefueron claves para momentos de reflexión, para pensar en nuevas soluciones de la pregunta central, diferentes a las que tenían al inicio de clase, además de transformar sus ideas previas, ya que se dieron cuenta de cómo funcionaba el órgano de la audición y de qué antecesor provenía su evolución, el cual se evidencio con la participación de ellos cuando se les hacía preguntas dirigidas a confirmar la transformación de sus ideas previas.

Por otro lado, la diversidad de actividades durante la planeación también fueron fundamentales para actuar sobre las dificultades de aprendizaje que se nombraron anteriormente, ya que se recalcó constantementecada concepto de manera que ellos fueran generándolos e interiorizándolos en sus mentes, pero de manera clara y sin posibles errores conceptuales que se generan cuando se enseñan y se aprenden estos temas.

\section{Conclusiones}

Es necesario tener cuidado para quienes deseen realizar una clase integrada mediante resolución de problemas en los temas de ciencias naturales, ya que pueden tener falencias al no saberredactar una pregunta problema, mostrando así preguntas cerradas, o que solo tienen solución mediante un algoritmo mecánico, o muy evidentes para los estudiantes.

Por otro lado, se evidenció con los resultados obtenidos, un aprendizaje en los estudiantes sobre el sentido de la audición, mediante resolución de problemas, sin embargo, para tener un buen resultado en la pregunta problema y en el aprendizaje del estudiante en general creemos que se debe de tener en cuenta una buena articulación en el plan de actividades antes de realizar clase, para que nutra de conceptos al estudiante antes de resolver la pregunta que se pretenda plantear.

Estapropuesta nos permitió como maestros guiar al estudiante a reflexionar sobre lapregunta centraly fue así como ellos a través del análisis que hicierona partir del dialogo permanente con el maestro facilitado por un plan de clase bien articulado llegarona dar una solución diferente a la inicial de la pregunta problema. , como también de superar los problemas de aprendizaje y de generar una transformación de sus ideas previas.

Para nosotros como maestros en formación la experiencia de la secuenciación de una clase en el marco de resolución de problemas, su preparación y aplicación nos brindó elementos teóricos y prácticos para abordar problemáticas 
Edición Extra-Ordinaria. ISSN 2027-1034 P. p 337-344

Memorias del VII Encuentro Nacional de Experiencias en la Enseñanza de la Biología y la Educación Ambiental y II Congreso Nacional de Investigación en la Enseñanza de la Biología

en aula en la enseñanza de las Ciencias Naturales requisito indispensable para nuestro trabajo de práctica profesional docente.

\section{Bibliografía}

Banet E. (2000), Enseñanza y aprendizaje del conocimiento de la biología. En: Cañal P. y Perales F. Didáctica de las ciencias experimentales (pp. 450 - 478); editorial Marfil S.A

Cheers G. (2008), Los oídos. En Cheers G. Anatómica-enciclopedia visual del cuerpo humano, tomo 9: oído y cuello (pp. 1 - 29), edición de producciones Cantabria SAC, primera edición.

Cornejo J. (2007), Formación integral docente en Ciencias Exactas y Naturales, Revista iberoamericana de educación, vol. 43, n5, 1 - 11.

Guanche A. (2005), La enseñanza problémica de las ciencias naturales, Revista iberoamericana de educación, vol. 36, n6, 1-21.

Quiroga J. (1975), Capítulo IV Acústica. En Quiroga J. Curso de enseñanza media de la física (pp. 52 - 64), editorial Bedout S.A., Medellín-Colombia, decima primera edición.

Saura O. y Pro A. (2000), Enseñanza y aprendizaje del conocimiento físico. En: Cañal P. y Perales F. Didáctica de las ciencias experimentales (pp. 389 - 420); editorial Marfil S.A 